The Astrophysical Journal, 448:L123-L126, 1995 August 1

(C) 1995. The American Astronomical Society. All rights reserved. Printed in U.S.A.

\title{
ANOMALOUSLY EXCITED OH AND COMPETITION BETWEEN MASER TRANSITIONS TOWARD CENTAURUS A
}

\author{
Huib JAN VAN LANGEVELDE \\ Joint Institute for VLBI in Europe, Radiosterrenwacht Dwingeloo, Postbus 2, 7990 AA Dwingeloo, The Netherlands ${ }^{1}$ \\ AND \\ Ewine F. van Dishoeck, Maartje N. Sevenster, and Frank P. Israel \\ Sterrewacht Leiden, P.O. Box 9513, 2300 RA Leiden, The Netherlands \\ Received 1995 April 21; accepted 1995 May 25
}

\begin{abstract}
The ground-state radio transitions of $\mathrm{OH}$ have been observed toward the nucleus of the nearby active galaxy Centaurus A, using the Australia Telescope Compact Array. The main lines at rest frequencies 1667 and 1665 $\mathrm{MHz}$ show molecular absorption at velocities similar to those observed for other molecular species at millimeter wavelengths, which indicates that the lines of sight at radio and millimeter wavelengths sample the same material. The satellite transitions at 1612 and $1720 \mathrm{MHz}$ exhibit stimulated emission and absorption, including the first extragalactic detection of maser emission in the $1720 \mathrm{MHz}$ line. A remarkable feature of the two satellite transitions is that they are conjugate for all velocity components, with one in emission and the other equally strong in absorption. This is explained as the competition of the two lines for the same inverting infrared radiation in the regime in which the infrared lines are just becoming optically thick. The transition from $1720 \mathrm{MHz}$ to 1612 $\mathrm{MHz}$ maser emission depends critically on the $\mathrm{OH}$ column density and therefore yields direct information on the distribution of molecular gas.
\end{abstract}

Subject headings: galaxies: individual (Centaurus A) — galaxies: nuclei — ISM: molecules — masers — molecular processes - radio lines: galaxies

\section{INTRODUCTION}

The radio lines of $\mathrm{OH}$ in astronomical sources almost never show the ratios expected for thermal equilibrium. This results from the fact that the $\mathrm{OH}$ rotational transitions have very high radiative transition probabilities, which implies that the levels become thermalized at much higher densities than normally occur in the interstellar medium. In addition, the level diagram for $\mathrm{OH}$ is complex, with two rotational ladders ${ }^{2} \Pi_{3 / 2}$ and ${ }^{2} \Pi_{1 / 2}$ in which all levels are quadruplets because of $\Lambda$-doubling and hyperfine splitting. The excitation of the hyperfine lines is intimately interwoven with the rotational lines and therefore seldom shows normal line ratios. Because these rotational transitions can readily be excited by collisions or pumped by farinfrared radiation, maser emission of $\mathrm{OH}$ naturally arises in the interstellar medium, in circumstellar shells, and-in the two main lines-from "megamaser regions" in distant active galaxies.

Centaurus A is the radio source associated with the nearest active galaxy, NGC 5128. Cen A is unique in that its nucleus is bright both at radio and millimeter wavelengths. The radio core can be studied with techniques ranging from single dishes to VLBI (Shaffer \& Schilizzi 1975; Meier et al. 1989), and a number of molecular species have been detected in pencilbeam absorption toward the radio nucleus and the jets (Gardner \& Whiteoak 1976a, b; Seaquist \& Bell 1986, 1990). At millimeter wavelengths, strong absorption in the lowest rotational transitions of various molecules such as $\mathrm{CO}$ and $\mathrm{HCO}^{+}$has been seen toward the nucleus over a range of $\gtrsim 60$ $\mathrm{km} \mathrm{s}^{-1}$ in velocity (Israel et al. 1990, 1991; Eckart et al. 1990). The CO lines even reveal a weak, very broad thermal emission feature, which is identified with a circumnuclear torus of

\footnotetext{
${ }^{1}$ Temporarily at NRAO Socorro, P.O. Box 0, Socorro NM 87801.
}

diameter 400-500 pc (Israel et al. 1991; Israel 1992; Rydbeck et al. 1993) and which has been resolved in the continuum at submillimeter wavelengths (Hawarden et al. 1993). The absorption close to the systemic velocity is thought to originate from molecular clouds in the circumnuclear torus, whereas blueshifted absorption probably arises in clouds at larger distances from the nucleus, for example, in the dust lane. Other features appear redshifted from the systemic velocity and are interpreted as molecular clouds closer to the nucleus, perhaps falling inward.

Strong, saturated atomic hydrogen absorption has also been detected in the same velocity range (Van der Hulst, Golish \& Hashick 1983; Van Gorkom et al. 1990), but no simple one-to-one correspondence between the positions and strengths of the $\mathrm{H}$ I and molecular components is found. This can be due to a different distribution of atomic and molecular gas or to the fact that the radio and millimeter continuum sources are on slightly different lines of sight. To distinguish between these possibilities, we obtained high spectral resolution absorption measurements of $\mathrm{OH}$, a molecule that is chemically related to $\mathrm{CO}$ and $\mathrm{HCO}^{+}$but whose transitions lie close in wavelength to that of $\mathrm{H} \mathrm{I}$.

\section{OBSERVATIONS}

The Australian Telescope Compact Array (ATCA) ${ }^{2}$ consists of six $22 \mathrm{~m}$ dishes on a $6 \mathrm{~km}$ east-west baseline. The powerful backend allows dual linear polarization observations with a 4 $\mathrm{MHz}$ bandwidth in 1024 channels $\left(0.6 \mathrm{~km} \mathrm{~s}^{-1}\right.$ resolution $)$. The "main lines" at 1667 and $1665 \mathrm{MHz}$ were observed on 1992 October 18 and 19, respectively. The data on the satellite lines

\footnotetext{
${ }^{2}$ The Australia Telescope Compact Array is operated by the Australia Telescope National Facility, CSIRO, as a National Research Facility.
} 


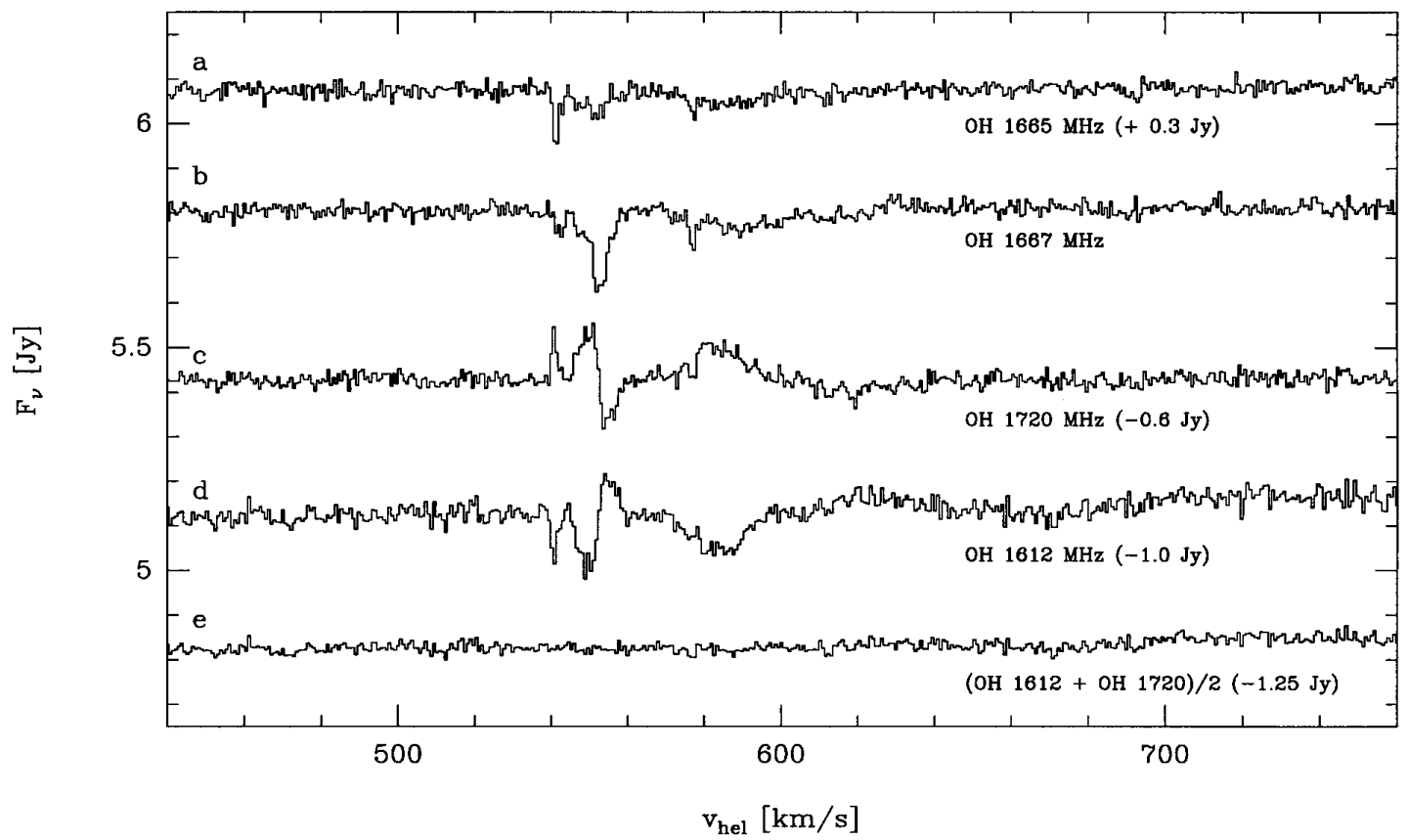

FIG. 1.- $(a-d)$ Spectra of the four OH lines against the nucleus of Centaurus A. The difference in the background level of the spectra is due to the change in core emission with observing frequency. (e) The sum of the 1612 and $1720 \mathrm{MHz}$ lines. This clearly demonstrates the competition for line inversion in these two transitions; there is no net emission left.

at 1720 and $1612 \mathrm{MHz}$ were obtained a year later on 1993 November 16 and 17.

The data were calibrated after conversion to Stokes $I$, using the standard calibrator $1934-638$, which also served as a bandpass calibrator. Phase calibration was obtained on 1320-466. The observations of the $1667 \mathrm{MHz}$ line, which had a central frequency of $1664 \mathrm{MHz}$ at the Cen A velocity, were affected by a narrow internal interference spike around $V_{\text {LSR }}=601 \mathrm{~km} \mathrm{~s}^{-1}$, the effects of which could be masked out. The data on the $1612 \mathrm{MHz}$ transition were of poor quality because of intermittent external interference at the observing frequency of $1609 \mathrm{MHz}$ by the Russian Glonass system. After careful flagging, the data are usable, but the low-level baseline problem in the $1612 \mathrm{MHz}$ spectrum can be attributed to this interference.

The $12 \mathrm{hr}$ synthesis produces maps with a typical resolving beam size of $7.3 \times 5.3$, but the image fidelity is limited because only a single ATCA configuration was used. However, besides the unresolved core $\left(\alpha, \delta[\mathrm{J} 2000]=13^{\mathrm{h}} 25^{\mathrm{m}} 27^{\mathrm{s}} .62\right.$, $-43^{\circ} 01^{\prime} 7^{\prime \prime}$. 8 ), the inner jet is easily distinguishable in maps of the continuum emission. In Figures $1 a-1 d$, the spectra against the core are shown for all four transitions. These spectra were taken from fully processed maps in order to make sure that the limited UV coverage does not affect their appearance.

\section{DISCUSSION}

\subsection{Radio versus Millimeter Absorption}

The 1665 and $1667 \mathrm{MHz}$ lines, presented in Figures $1 a$ and $1 b$, show absorption from several components. The $1667 \mathrm{MHz}$ line was previously detected in this source (Gardner \& Whiteoak 1976a, b; Seaquist \& Bell 1990), but the spectral resolution of these observations was too low to resolve it into several components. The high spectral resolution and sensitiv- ity of ATCA has allowed detection of the $1665 \mathrm{MHz}$ line in this galaxy for the first time.

The systemic velocity of Cen $\mathrm{A}$ is $V_{\mathrm{LSR}} \approx 550 \mathrm{~km} \mathrm{~s}^{-1}$, and the deepest $1667 \mathrm{MHz}$ feature is observed at this velocity. This feature has also been seen in other molecules and is identified with gas in the circumnuclear torus. The $1665 \mathrm{MHz}$ line occurs at this velocity as well but also shows strong absorption slightly blueshifted from the main component at $V_{\mathrm{LSR}}=541 \mathrm{~km} \mathrm{~s}^{-1}$, which probably originates in molecular clouds in the dust lane. Both transitions show broad, shallow absorption at redshifted velocities, with some narrow absorption features superposed on the broad component, especially at $V_{\mathrm{LSR}}=577 \mathrm{~km} \mathrm{~s}^{-1}$. A comparison among the $\mathrm{OH}, \mathrm{HCO}^{+}$, and $\mathrm{CO}$ spectra shows that there is a good one-to-one correspondence of the different absorption features, both at blueshifted and redshifted velocities, in spite of the different observing wavelengths. Thus, the poor correspondence between the $\mathrm{H}$ I and the molecular absorption features is not due to different lines of sight but must result from different physical and chemical conditions in the various components. In particular, the blueshifted clouds in the dust lane are very poor in atomic hydrogen, whereas the redshifted cloud at $V_{\mathrm{LSR}}=584 \mathrm{~km} \mathrm{~s}^{-1}$ has a comparatively low molecular content.

\section{2. $\mathrm{OH}$ Excitation}

As is common for $\mathrm{OH}$, the main-line transitions show anomalous excitation in all velocity components. The 1667 $\mathrm{MHz} / 1665 \mathrm{MHz}$ optical depth ratios vary from 1.9 for the systemic velocity component to 0.7 for the blueshifted absorption, whereas the LTE ratio is 1.8. In order to study the excitation properties further, the 1612 and $1720 \mathrm{MHz}$ transitions were observed as well (Figs. $1 c$ and $1 d$ ); they show strong absorption and emission in all components. The small resolving beam of the ATCA and the high background emission 
imply a very high brightness temperature in the emission features $(\approx 20,000 \mathrm{~K})$. Thus, although the (beam-averaged) optical depths are quite modest, both transitions show maser emission. This is the first clear extragalactic detection of maser emission in the $1720 \mathrm{MHz}$ line, although the detection of 1720 $\mathrm{MHz}$ emission by Turner (1985) in NGC 253 is most likely caused by maser action as well.

A remarkable property of the two satellite lines is the fact that they show exactly conjugate behavior. For the $550 \mathrm{~km} \mathrm{~s}^{-1}$ component, strong absorption is seen in the $1667 \mathrm{MHz}$ line, but the 1612 and $1720 \mathrm{MHz}$ lines "flip" right across this feature. This behavior is most clearly demonstrated by adding the two profiles, yielding a spectrum that is featureless within the noise limits (Fig. 1e). This implies that the optical depth in the satellite lines has the same absolute value to $\approx 10 \%$ accuracy.

The physical explanation for this phenomenon is the fact that the two transitions compete for the same pumping photons (Elitzur 1993, § 9.1). The most important transitions for the excitation of the hyperfine levels in the ground state are the rotational transitions connected with the first excited states in both ladders, the ${ }^{2} \Pi_{3 / 2} J=5 / 2 \rightarrow{ }^{2} \Pi_{3 / 2} J=3 / 2$ intraladder transition at $119 \mu \mathrm{m}$ and the ${ }^{2} \Pi_{1 / 2} J=1 / 2 \rightarrow{ }^{2} \Pi_{3 / 2} J=3 / 2$ cross-ladder transition at $79 \mu \mathrm{m}$. Any excitation of the $\mathrm{OH}$ molecule will cascade back to the ground state through either of these far-infrared lines. The selection rules are such that parity must change and only $|\Delta F|=1,0$ is allowed. In case the radiative decay is optically thick, only the number of connections dictates the populations in the ground-state levels but not the individual transition probabilities. Intraladder pumping followed by a cascade to the ground state through the ${ }^{2} \Pi_{3 / 2}$ $J=5 / 2$ level therefore naturally leads to an overpopulated $F=2$ level, which results in stimulated emission in the 1720 $\mathrm{MHz}$ line and stimulated absorption in the $1612 \mathrm{MHz}$ line. In case the pumping is cross-ladder, the route through ${ }^{2} \Pi_{1 / 2}$ $J=1 / 2$ leads exactly to the opposite: the $F=1$ level is overpopulated, which gives rise to $1612 \mathrm{MHz}$ emission and $1720 \mathrm{MHz}$ absorption. Which route is more important depends on the details of the pumping, but in case both ladders are excited, it is argued that stimulated emission in the 1612 $\mathrm{MHz}$ line will dominate because of the different statistical weights of the two routes (Elitzur 1993, p. 234). This scheme can provide the conjugate spectra, and such behavior has been known for some time in a qualitative sense for Galactic interstellar clouds (Goss 1968; Goss et al. 1973; Caswell \& Haynes 1975).

The above mechanism assumes that the rotational lines are optically thick. Because the Einstein coefficient for the intraladder ${ }^{2} \Pi_{3 / 2} J=5 / 2 \rightarrow{ }^{2} \Pi_{3 / 2} J=3 / 2$ transition is nearly an order of magnitude larger than that of the cross-ladder ${ }^{2} \Pi_{1 / 2}$ $J=1 / 2 \rightarrow{ }^{2} \Pi_{3 / 2} J=3 / 2$ transition, the intraladder line will become optically thick for smaller column densities than the cross-ladder line. Roughly, this occurs around $N_{\mathrm{OH}} /$ $\Delta V \approx 1 \times 10^{14} \mathrm{~cm}^{-2} \mathrm{~km}^{-1} \mathrm{~s}$ for the intraladder $(1720 \mathrm{MHz}$ pumping) and $N_{\mathrm{OH}} / \Delta V \approx 9 \times 10^{14} \mathrm{~cm}^{-2} \mathrm{~km}^{-1} \mathrm{~s}$ for the crossladder (1612 MHz pumping) lines.

We carried out non-LTE excitation calculations of $\mathrm{OH}$ to confirm this interpretation and to determine whether it is possible to explain the excitation of the different components in a physical model. Such computations involve all allowed radiative transitions between all $\mathrm{OH}$ hyperfine levels up to 415 $\mathrm{K}$ above the ground state. For collisional (de-)excitation by para- and ortho- $\mathrm{H}_{2}$, rates between the $\mathrm{OH}$ hyperfine levels were used, which have recently become available for the first time from theoretical calculations by Offer, Van Hemert, \& Van Dishoeck (1994). In fact, by using these cross sections after the 1992 observations of the main lines, masing in the satellite lines was predicted and subsequently confirmed by the 1993 observations.

The radiative transfer is treated using an escape probability approach for a homogeneous medium, corresponding to individual clouds in Cen A. In cases like this in which the total gain in the lines is modest, the adopted escape probability method should be a safe approach. Input parameters include the $\mathrm{H}_{2}$ density and temperature and the $\mathrm{OH}$ column density, which controls the optical depth in the infrared pumping lines. The ambient radiation field is also of importance, because the rotational lines that initiate the pumping occur at 119, 79, 53, and $35 \mu \mathrm{m}$. Like most galaxies, the far-infrared part of the radiation will be dominated by emission from cold $T_{\text {dust }} \approx 20-$ 50 K dust (e.g., Knapp, Bies \& Van Gorkom 1990). For Cen A, this emission is resolved along the "dusty disk" in the outer parts of the galaxy (Marston \& Dickens 1988), but there is also a component due to the circumnuclear disk (Joy et al. 1988; Israel et al. 1990; Hawarden et al. 1993). There is clear evidence for excess 25 and $12 \mu \mathrm{m}$ emission along the disk (Marston \& Dickens 1988). Moreover, centimeter radiation, for instance from the VLBI core, can affect the excitation within the $\Lambda$-doublets of the ground state.

A large number of non-LTE models were run to explore the effects of density, column density, gas temperature, and radiation field on the four transitions. Figure 2 shows the result of one such set of model calculations for which the molecular gas in the torus at $300 \mathrm{pc}$ from the nucleus was modeled. There is a distinct region in Figure 2 in which the 1612 and $1720 \mathrm{MHz}$ lines clearly show the opposite behavior. Since these calculations were carried out for $\Delta V=1 \mathrm{~km} \mathrm{~s}^{-1}$, the "flip" occurs at $N_{\mathrm{OH}} \approx 1 \times 10^{15} \mathrm{~cm}^{-2}$, where the cross-ladder rotational lines become optically thick. The parameters of the model do influence the excitation, and not every set of parameters yields maser emission in both satellite lines. However, in all cases that masing in both lines does occur, the location of this boundary was found to be at $N_{\mathrm{OH}} / \Delta V \approx 1 \times 10^{15} \mathrm{~cm}^{-2} \mathrm{~km}^{-1} \mathrm{~s}$.

We conclude that the difference in the excitation of the satellite lines depends indeed primarily on the column density of $\mathrm{OH}$. This explains that the effect of conjugate satellite lines is observed for all components, even though different physical conditions may prevail in each of these. The conjugate behavior of satellite lines can also be obtained for conditions that resemble those in the "normal" interstellar medium in NGC 5128. Some additional constraints on the physical conditions can be obtained from the main lines. For instance, a deep 1665 $\mathrm{MHz}$ absorption with little $1667 \mathrm{MHz}$ can, according to simulations, occur in a low-density environment. It therefore seems natural to identify the blueshifted feature with a lowdensity $n \lesssim 10^{4} \mathrm{~cm}^{-3}$ and small column density $\left(N_{\mathrm{OH}} \lesssim 10^{14}\right.$ $\mathrm{cm}^{-2}$ ) cloud in the outer dust lane of Cen A. The redshifted spectrum, on the other hand, shows a strong feature in the satellite lines, while the main lines show a near-thermal ratio. Such behavior could occur at higher densities, $n \gtrsim 10^{6} \mathrm{~cm}^{-3}$. However, the interpretation of the main lines depends on the local radiation field, of which we have limited knowledge.

It was found that the emission from the radio nucleus can influence the excitation of the $\Lambda$-doublets in the ground state 

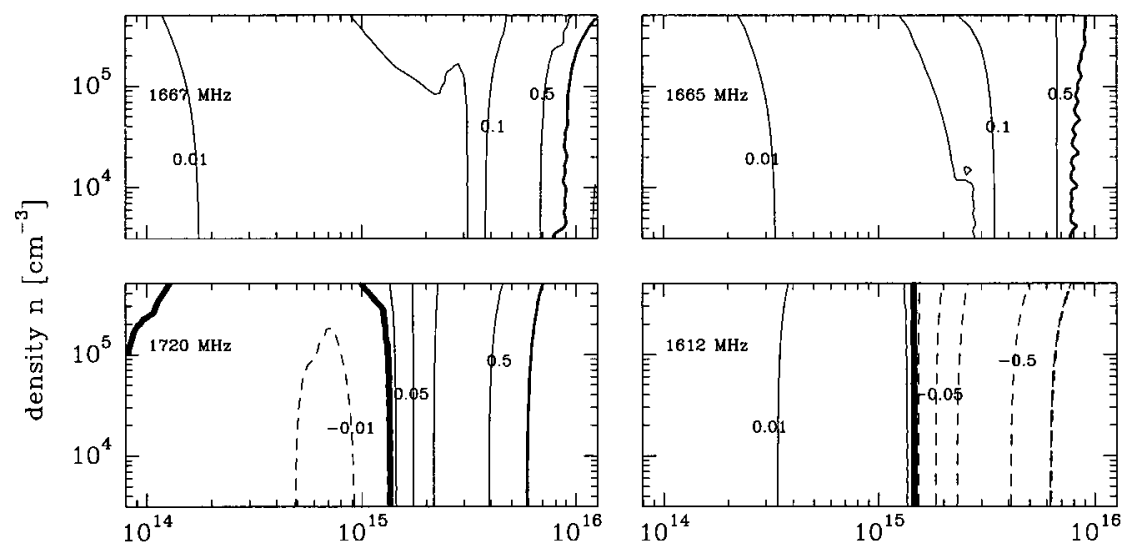

column density $\mathcal{N}\left[\mathrm{cm}^{-2}\right]$

FIG. 2.-Excitation of the four $\mathrm{OH}$ lines as a function of density and column density. Solid lines are positive optical depth; dashed lines are negative (maser) optical depth. Contours are $\tau= \pm 0.01,0.05,0.1,0.5,1$, and 5 . The model is characterized by two IR components, both with $\beta=1$ and $\left.T_{1}=43 \mathrm{~K}, E(B-V)\right)_{1}=10$, and $\Delta \Omega_{1}=1$ and $T_{2}=150 \mathrm{~K}, E(B-V)_{2}=1$, and $\Delta \Omega_{2}=0.1$, respectively, as well as a radio component with $\alpha=0.2 \mathrm{between} 1 \mathrm{and} 500 \mathrm{GHz}, I_{1} \mathrm{GHz}=10^{-9}$ ergs $\mathrm{cm}^{-2} \mathrm{~Hz}^{-1}$, and $\Delta \Omega_{\text {radio }}=10^{-4}$. The collision partner was $\mathrm{H}_{2}$ with $T_{\text {kin }}=10 \mathrm{~K}$ and an ortho-para ratio of 0.2 .

out to $1 \mathrm{kpc}$. Especially within $100 \mathrm{pc}$, the excitation temperatures are substantially increased because the molecules attempt to match the brightness temperature of the radiation field. Furthermore, we find that a weak mid-infrared component is necessary to excite the 1612 and 1720 inversions in our model through absorption in the $35 \mu \mathrm{m}$ line, although the temperature and density in the torus are in principle sufficient to excite $\mathrm{OH}$ collisionally to the first rotational levels.

The 1612/1720 MHz "flip" is observed to occur right across the $550 \mathrm{~km} \mathrm{~s}^{-1}$ component as well as the broad redshifted component. This implies that the column density for the 550 $\mathrm{km} \mathrm{s}^{-1}$ component can be derived without further assumptions: $N_{\mathrm{OH}} \approx 6 \times 10^{15} \mathrm{~cm}^{-2}$ for the measured line width $\Delta V=6 \mathrm{~km} \mathrm{~s}^{-1}$. The total $\mathrm{H}_{2}$ column density in the main component at $V_{\mathrm{LSR}}=550 \mathrm{~km} \mathrm{~s}^{-1}$ is estimated to be $N_{\mathrm{H}_{2}}=(2 \pm 1) \times 10^{22} \mathrm{~cm}^{-2}$ (Israel et al. 1991), which yields an $\mathrm{OH}$ abundance of $(2-6) \times 10^{-7}$. The optical depth of the main absorption feature in the $1667 \mathrm{MHz}$ line is consistent with this column density, as can be inferred from Figure 2. This abundance is similar to, or even slightly higher than, typical
$\mathrm{OH}$ abundances derived for molecular clouds in our Galaxy (Irvine et al. 1987; Colgan, Salpeter, \& Terzian 1989).

If we assume that the hydroxyl lines arise from the same molecular clouds as the millimeter absorption features, then typically $n \gtrsim 10^{4} \mathrm{~cm}^{-3}$. For an $\mathrm{OH}$ abundance of $3 \times 10^{-7} \mathrm{a}$ cloud size of $\lesssim 1 \mathrm{pc}$ is derived. This number is probably uncertain by an order of magnitude, but it seems inconsistent with a homogeneous torus. For the shallow redshifted component, a similar calculation would yield high densities $n \approx 10^{6}$ $\mathrm{cm}^{-3}$ for parsec-scale clouds because this component has such large velocity width. This is in agreement with the high density and high temperatures that can be inferred for the redshifted features from $\mathrm{CO} J=3 \rightarrow 2$ absorption and $\mathrm{HCN} / \mathrm{HNC}$ ratios (Eckart et al. 1990; Israel et al. 1991).

The authors would like to thank the ATCA staff, especially Jessica Chapman, Neil Killeen, and Mark Wieringa, for help with the observations and Alison Offer for providing the $\mathrm{OH}-\mathrm{H}_{2}$ rates. This work was supported by the Netherlands Organization for Scientific Research (NWO).
Caswell, J. L., \& Haynes, R. F. 1975, MNRAS, 173, 649

Colgan, S. W. J., Salpeter, E. E., \& Terzian, Y. 1989, ApJ, 336, 231

Eckart, A., Cameron, M., Genzel, R., Jackson, J. M., Rothermel, H., Stutzki, J., Rydbeck, G., \& Wiklind, T. 1990, ApJ, 365, 522

Elitzur, M. 1993, Astronomical Masers (Dordrecht: Kluwer)

Gardner, F. F., \& Whiteoak, J. B. 1976a, Proc. Astron. Soc. Australia, 3, 63 1976b, MNRAS, 175, 9P

Goss, W. M. 1968, ApJS, 15, 131

Goss, W. M., Johansson, L. E. B., Elldér, J., Höglund, B., Nguyen-Q-Rieu, \& Winnberg, A. 1973, A\&A, 28, 89

Hawarden, T. G., Sandell, G., Matthews, H. E., Friberg, P., Watt, G. D., \& Smith P. A. 1993, MNRAS, 260, 844

Irvine, W. M., Goldsmith, P. F., \& Hjalmarson, Å. 1987, in Interstellar Processes, ed. D. Hollenbach \& H. A. Thronson (Dordrecht: Reidel), 561 Israel, F. P. 1992, A\&A, 265, 487

Israel, F. P., van Dishoeck, E. F., Baas, F., de Graauw, T., \& Phillips, T. G. 1991, A\&A, 245, L13

\section{REFERENCES}

Israel, F. P., van Dishoeck, E. F., Baas, F., Koornneef, J., Black, J. H., \& de Graauw, T. 1990, A\&A, 227, 342

Joy, M., Lester, D. F., Harvey, P. M., \& Ellis, H. B. 1988, ApJ, 326, 662

Knapp, G. R., Bies, W. E., \& Van Gorkom, J. H. 1990, AJ, 99, 476

Marston, A. P., \& Dickens, R. J. 1988, A\&A, 193, 27

Meier, D. L., et al. 1989, AJ, 98, 27

Offer, A. R., Van Hemert, M. C., \& van Dishoeck, E. F. 1994, J. Chem. Phys., 100,362

Rydbeck, G., Wiklind, T., Cameron, M., Wild, W., Eckart, A., Genzel, R., \& Rothermel, H. 1993, A\&A, 270, L13

Seaquist, E. R., \& Bell, M. B. 1986, ApJ, 303, L67

. 1990, ApJ, 364, L94

Shaffer, D. B., \& Schilizzi, R. T. 1975, AJ, 80, 573

Turner, B. E. 1985, ApJ, 299, 312

Van der Hulst, J. M., Golish, W. F., \& Hashick, A. D. 1983, ApJ, 264, L37

Van Gorkom, J. H., Van der Hulst, J. M., Haschick, A. D., \& Tubbs, A. D. 1990, AJ, 99, 1781 